\title{
Neural activity during simple visual imagery compared with mental rotation imagery in young adults with smartphone overuse
}

\author{
Sujin Hwang ${ }^{a}$, Jeong-Weon Lee ${ }^{b}$, Si-Nae Ahn ${ }^{b}$ \\ aDepartment of Physical Therapy, Division of Health Science, Baekseok University, Cheonan, Republic of Korea \\ ${ }^{b}$ Department of Occupational Therapy, Yeoju Institute of Technology, Yeoju, Republic of Korea
}

Objective: This research investigated the effects of simple visual imagery and mental rotation imagery on neural activity of adults who are at high risk of smart phone addiction by measuring their electroencephalography (EEG).

Design: Cross-sectional study.

Methods: Thirty people with a high risk of smart phone addiction was selected and then were evaluated for their neural activation patterns using EEG after reminding them about simple visual imagery and mental rotation imagery. A simple visual image was applied for 20 seconds using a smartphone. This was followed by a resting period of 20 seconds. Mental rotation imagery was applied for 20 seconds. During mental rotation imagery, the rotational angle was selected at random. We compared activation patterns according to the analyzed EEG with hemisphere reminding them about imagery.

Results: On the EEG, theta rhythm from the left hemisphere parietal area increased when the subjects were reminded of mental rotation imagery, and sensorimotor rhythm from close to the left hemisphere area increased when the subjects were reminded of simple visual imagery.

Conclusions: Neural activation from the left hemisphere occurs for motor imagery in adults who are at high risk of smart phone addiction. These results identify a neural mechanism of adults who a have high risk of smart phone addiction, which may provide contribute to the development of motor rehabilitation for smartphone users.

Key Words: Electroencephalography, Imagery, Smart phone

\section{Introduction}

In the past decade, there has been a rapid increase in the use of mobile devices, particularly smartphones. Smartphones became widespread in the late 2000. The Japanese firm NTT DoCoMo released the first smartphone in 1999. The international data corporation reported that there are over 1.5 billion smartphone users around the world, and it has been estimated that more than 1 billion smartphones would be sold in 2016 [1]. In the United States, 56\% of adults are now smartphone owners, while in 2012, smartphone owners made up $79 \%$ of the population in Switzerland [1]. Park [2] reported that, in Korea, $58 \%$ of adults owned smartphones, while $84 \%$ of college students used smartphones in 2011. Extensive use of smartphones can be associated with physical health-related problems, psychological problems, and social problems. Smartphone addiction has been defined as the overuse of smartphones to the extent that it disturbs the users' daily lives [3].

The core features of smartphone overuse include sustained engagement in a behavior despite its negative effects, decreased control over participation in the behavior, compulsive participation, and appetitive or craving urges that instantly precede engagement in the behavior [4]. Roberts and

Received: 3 November, 2017 Revised: 25 November, 2017 Accepted: 26 November, 2017

Corresponding author: Sujin Hwang

Department of Physical Therapy, Division of Health Science, Baekseok University, 76 Munam-ro, Dongnam-gu, Cheonan 31065 , Republic of Korea Tel: 82-41-550-2309 Fax: 82-41-550-2829 E-mail: sujin928@gmail.com

(c) This is an Open-Access article distributed under the terms of the Creative Commons Attribution Non-Commercial License (http://creativecommons.org/licenses/ by-nc/4.0) which permits unrestricted non-commercial use, distribution, and reproduction in any medium, provided the original work is properly cited.

Copyright $@ 2017$ Korean Academy of Physical Therapy Rehabilitation Science 
colleagues [5] found that college students spent almost 9 hours per day on their cell phones. Notably, adults often have different schedules to adolescents, with the luxury to determine their own use of electronic media, freedom from parent-imposed constraints. These factors may cause higher use of smartphone or electronic media devices among young adults, such as university students [5].

Prolonged smartphone use could cause a decrease in overall physical functional activities as well as the development of primary problems, such as problems with vision and hearing, and secondary problems, such as problems with visual perceptive function, visual memory, and cognitive function $[3,6]$. In particular, smartphone overuse alters the visual images associated with visuoperceptual and cognitive function during the use of a smartphone [3,5]. The typical method used to change the visual image of the brain is to make the required comparison. Participants imagine one object to be rotated into the same orientation as another object. This method is termed mental rotation and is a function of visual representation in the human brain. Mental rotation makes the brain move objects in order to help understand what they are and where they belong. The aim is to rotate the image without requiring the use of higher cognitive function and attention and to think of simple visual images to further promote the activation of the brain [7-11].

Mental rotation performance is impaired in overweight children, due to a reduction in visuospatial organization. Subclinical neck pain may impair the ability to perform a complex mental rotation task involving cerebellar connections, possibly due to an altered body schema $[6,9]$. However, previous studies have not measured brain activation during investigations of differences in simple visual imagery and mental rotation imagery with regard to smartphone overuse [12]. To verify the differences between internal memory and attention following excessive exposure to smartphones, this study compared neural activity during simple visual imagery and mental rotation imagery in young adults who overuse smartphones. This study hypothesized that there would be a significant difference in neural activity during simple visual imagery and mental rotation imagery, and that neural activation during simple visual imagery and mental rotation imagery would be altered in participants who are at high risk of smart phone addiction.
Table 1. General characteristics of the participants $\quad(\mathrm{N}=30)$

\begin{tabular}{lc}
\hline \multicolumn{1}{c}{ Characteristic } & Data \\
\hline Sex & \\
Male & $17(56.7)$ \\
Female & $13(43.3)$ \\
Age $(\mathrm{yr})$ & $23.6 \pm 2.5(20.0-29.0)$ \\
Height $(\mathrm{cm})$ & $171.1 \pm 7.5(160.0-185.0)$ \\
Weight $(\mathrm{kg})$ & $65.5 \pm 9.3(51.0-83.0)$ \\
SAPSA & $45.1 \pm 1.3(42.0-49.0)$ \\
\hline
\end{tabular}

Values are presented as number (\%) or mean $\pm \mathrm{SD}$ (range). SAPSA: smart-phone addiction proneness scale for adult.

\section{Methods}

\section{Participants}

Thirty people at high risk of mobile phone dependence syndrome were selected in this study. The inclusion criteria were as follows: (1) smartphone ownership; (2) a score of 44 points or over in the smartphone addiction proneness scale for adults; and (3) spontaneous consent. Individuals were excluded from this study if they met the following exclusion criteria for the EEG through participant self-reporting: (1) epilepsy; (2) mental disorders; and (3) sensory impairments. Before participating in the study, all participants gave consent, according to guidelines of the Institutional Review Board of the Baekseok University (IRB No. BUIRB-201608HR-023). Table 1 shows the clinical and general characteristics of the participants.

\section{Procedure}

This cross-sectional study was designed to investigate the difference in neural activation during simple visual imagery and mental rotation imagery in young adults with smartphone dependence syndrome (Figure 1). The visual information used to produce the imagery processing in this study was provided by a geometric figure on a smartphone (Figure 2). The experimental process was conducted in a calm and well-organized room. To minimize any external bias during the experimental process, the participants were asked to maintain a fixed seated position with closed eyes until the experiment was completed. The participants conducted simple visual imagery and mental rotation imagery. Neural activation patterns were measured using electroencephalography (EEG).

For the first 3 minutes, this study measured the participants' background EEG. A simple visual image was then applied for 20 seconds using a smartphone. This was fol- 


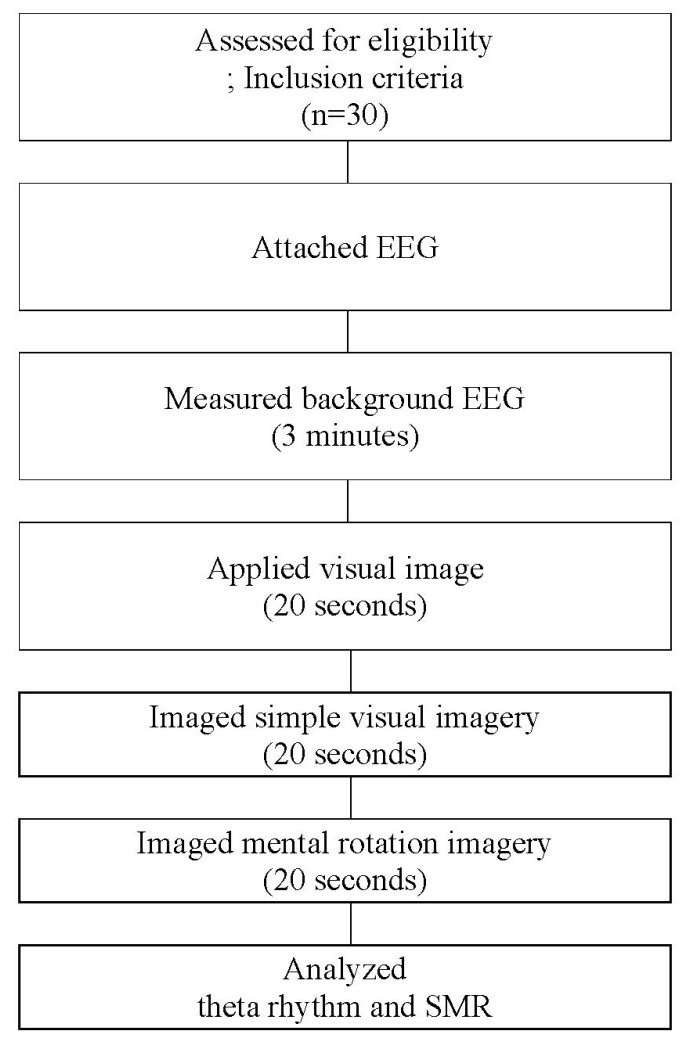

Figure 1. The study paradigm. EEG: electroencephalography, SMR: sensory motor rhythm.

lowed by a resting period of 20 seconds. Finally, this study applied mental rotation imagery with closed eyes for 20 seconds. During mental rotation imagery, the rotational angle was selected at random. Neural activations were recorded from both hemispheres using EEG. Corrected data were compared to both hemispheres.

\section{Tasks}

This study measured neural activation patterns during simple visual imagery and mental rotation imagery. Simple visual imagery is a mental image that is similar to visual perception. Mental rotation imagery is a method used to examine a participants' awareness of objects in a three-dimensional environment. Mental rotation is a genuine transformation process, in which a shape is represented as passing through intermediate orientations before reaching the target orientation. This imagery has a positive effect on physical activity training [13]. This study used 2 types of visual imagery for evaluating neural activation differences.

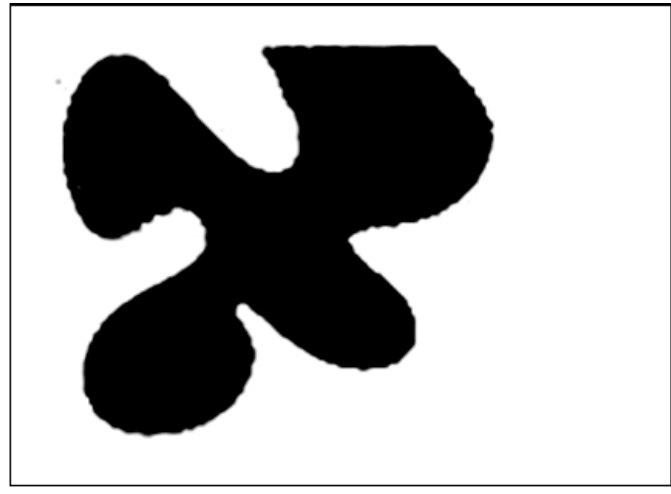

Figure 2. Geometric figure for visual image.

\section{Experimental equipment}

To determine the neurological changes in the brain, this study measured the brain waves of the participants using the CANS 3000 QEEG-8 (LAXTHA Inc., Daejeon, Korea). EEG was recorded using $\mathrm{Ag} / \mathrm{AgCl}$ cup electrodes attached to the scalp at 8 active sites (Fp1, Fp2, C3, C4, P3, P4, T3, and T4), according to the international 10/20 system. In addition, a relative power analysis was conducted on the values at the 8 active positions and the participants' theta rhythm and sensory motor rhythm (SMR) were analyzed to compare the effects of simple visual imagery and mental rotation imagery.

\section{Data analysis}

For the EEG analysis, this study collected data on the relative power of each participants' theta rhythm and SMR. A comparison of the relative power spectrum calculations was conducted using the research task conditions and the fast fourier transform. The theta rhythm and SMR were determined using the A1 and A2 sites as the standard electrodes. For statistical analysis, this study conducted a descriptive analysis and paired t-test using PASW Statistics ver. 18.0 (IBM Co., Armonk, NY, USA). Significance was set at $p<0.05$.

\section{Results}

This study measured the relative power of the theta rhythm and the SMR in the prefrontal, central, parietal, and temporal lobes of both hemispheres to compare the effects of simple visual imagery and mental rotation imagery (Tables 2, 3). In participants who were at high risk of smart phone addiction, mental rotation imagery induced higher 
Table 2. Value of theta rhythm during imagery

$(\mathrm{N}=30)$

\begin{tabular}{clccc}
\hline Electroencephalography area & Simple imagery & Mental rotation imagery & $\mathrm{z}(p)$ \\
\hline \multirow{2}{*}{ Right } & Prefrontal & $0.103(0.052)$ & $0.107(0.044)$ & $-0.478(0.637)$ \\
& Central & $0.126(0.051)$ & $0.129(0.049)$ & $-0.338(0.738)$ \\
& Parietal & $0.103(0.047)$ & $0.108(0.050)$ & $-0.514(0.612)$ \\
\multirow{2}{*}{ Left } & Temporal & $0.089(0.036)$ & $0.101(0.037)$ & $-1.752(0.091)$ \\
& Prefrontal & $0.103(0.051)$ & $0.108(0.046)$ & $-0.537(0.596)$ \\
& Central & $0.124(0.052)$ & $0.131(0.049)$ & $-0.725(0.475)$ \\
& Parietal & $0.097(0.052)$ & $0.119(0.041)$ & $-2.214(0.035)$ \\
\hline
\end{tabular}

Values are presented as mean (SD).

Table 3. Value of sensory motor rhythm during imagery

$(\mathrm{N}=30)$

\begin{tabular}{clccr}
\hline \multirow{2}{*}{ Electroencephalography area } & Simple imagery & Mental rotation imagery & $\mathrm{z}(p)$ \\
\hline \multirow{2}{*}{ Right } & Prefrontal & $0.032(0.026)$ & $0.032(0.026)$ & $-0.018(0.986)$ \\
& Central & $0.054(0.040)$ & $0.056(0.03)$ & $-0.534(0.597)$ \\
& Parietal & $0.060(0.048)$ & $0.063(0.046)$ & $-0.387(0.702)$ \\
\multirow{3}{*}{ Left } & Temporal & $0.083(0.063)$ & $0.084(0.062)$ & $-0.100(0.921)$ \\
& Prefrontal & $0.050(0.061)$ & $0.028(0.028)$ & $2.154(0.040)$ \\
& Central & $0.059(0.052)$ & $0.044(0.024)$ & $1.778(0.087)$ \\
& Parietal & $0.066(0.057)$ & $0.043(0.021)$ & $2.396(0.024)$ \\
& Temporal & $0.081(0.052)$ & $0.075(0.043)$ & $0.665(0.512)$ \\
\hline
\end{tabular}

Values are presented as mean (SD).

relative power of the theta rhythm in the prefrontal, central, parietal and temporal areas of the left hemisphere compared to the relative power recorded during simple visual imagery. The relative power of the theta rhythm was significantly higher only in the parietal area of the left hemisphere during mental rotation imagery compared to the relative power during simple visual imagery. However, there was no significant difference between activation during simple visual imagery and mental rotation imagery in the right hemispheres.

In the participants who were at high risk of smart phone addiction, simple visual imagery induced a greater relative power of the SMR at all four sites, including the prefrontal, central, parietal and temporal areas of the left hemisphere, compared to the relative power during mental rotation imagery. However, during mental rotation imagery, the relative power of the SMR was significantly higher in the prefrontal and parietal areas of the left hemisphere. Although mental rotation imagery produced a greater relative power of the SMR in the right hemisphere compared to the relative power during simple visual imagery, there was no significant difference between the two types of imagery at any of the four sites.

\section{Discussion}

This study investigated the effects of simple visual imagery and mental rotation imagery on neural activities in young adults who are at high risk of smart phone addiction through EEG. Thirty people who were at high risk of smart phone addiction were evaluated using EEG during simple visual imagery and mental rotation imagery. We compared the activation patterns between hemispheres using EEG. The theta rhythm from the left hemisphere parietal area increased during mental rotation imagery, and the SMR from the prefrontal and parietal areas in the left hemisphere increased during simple visual imagery.

Previously, neuroimaging studies have recorded activity during mental rotation tasks in areas of the frontal cortex, superior parietal cortex, and precentral cortex that are associated with motor planning and execution, spatial cognition, and motor simulation [13-16]. Other studies using the mental rotation task have recorded activation in the superior parietal lobule and sometimes the visual-perceptual areas [17-20]. Most studies have observed activation in the posterior parietal cortex during the mental rotation task. The posterior parietal cortex is thought to be involved in spatial 
processing and spatial attention. In agreement with previous studies, this study observed a difference in the relative power of the theta rhythm and the SMR in the parietal area between simple visual imagery and mental rotation imagery. However, in the frontal and precentral cortex, there was no difference in the relative power of the theta rhythm or the SMR between simple visual imagery and mental rotation imagery in adults who are at high risk of smart phone addiction.

A previous EEG study demonstrated that beta-band EEG power in the left frontal and bilateral parietal sites was suppressed during mental rotation [21]. According to the results of this study, mental rotation imagery facilitated an increase of the theta rhythm, while simple visual imagery facilitated an increase of the beta rhythm. Beta waves are produced during external attention, whilst theta waves are dominant in internal attention [22]. The results of this study identified an increase in the SMR from the prefrontal and parietal areas in the left hemisphere during simple visual imagery compared to mental rotation imagery in participants who overuse smartphones. However, the results of this study suggest that smartphone overuse facilitates external attention in visual imagery, thus, the results of this study contradict the results of previous studies.

Neural activations in frontal areas should be produced by mental rotation imagery during the presentation of geometric figures because of the neural functions of the frontal lobe such as attention, planning, organizing, problem solving and mental flexibility $[16,23]$. In normal adults, during mental rotation, neural activations are seen in the frontal cortex, the superior parietal cortex, and the precentral cortex. However, in this study, mental rotation imagery resulted in increased activation in the parietal cortex compared to simple visual imagery in young adults who overuse smartphones. The results of this study suggest that neural activation patterns are different between normal adults and adults who overuse smartphones.

In summary, this study confirmed that brain activation during simple visual imagery and mental rotation imagery is different in adults who overuse smartphones. There was a meaningful difference between theta rhythm and the SMR in this research because of the relationship between neural activation patterns and attention of visual imagery. In future studies, therapeutic approaches that use mental rotation imagery should incorporate brain measurements, such as EEG, to provide more evidence for the role of mental rotation imagery in daily activities in training by occupational therapists. This study has several limitations. This study did not measure any subjective or behavioral measures after each trial of simple visual imagery and mental rotation imagery, thus, there may be a high variability in the level of attention among the participants.

\section{Conflict of Interest}

The authors declared no potential conflicts of interest with respect to the authorship and/or publication of this article.

\section{References}

1. Demirci K, Akgönül M, Akpinar A. Relationship of smartphone use severity with sleep quality, depression, and anxiety in university students. J Behav Addict 2015;4:85-92.

2. Park YM. A study on adults' smartphone addiction and mental health [MA dissertation]. Wonju: Sangji University; 2011.

3. Griffiths MD, Kuss DJ, Billieux J, Pontes HM. The evolution of internet addiction: a global perspective. Addict Behav 2016;53: 193-5.

4. Mok JY, Choi SW, Kim DJ, Choi JS, Lee J, Ahn H, et al. Latent class analysis on internet and smartphone addiction in college students. Neuropsychiatr Dis Treat 2014;10:817-28.

5. Roberts JA, Yaya LH, Manolis C. The invisible addiction: cell-phone activities and addiction among male and female college students. J Behav Addict 2014;3:254-65.

6. Lee KE, Kim SH, Ha TY, Yoo YM, Han JJ, Jung JH, et al. Dependency on smartphone use and its association with anxiety in Korea. Public Health Rep 2016;131:411-9.

7. Yahagi S, Shimura K, Kasai T. An increase in cortical excitability with no change in spinal excitability during motor imagery. Percept Mot Skills 1996;83:288-90.

8. Suzuki T, Bunno Y, Onigata C, Tani M, Uragami S. Excitability of spinal neural function by motor imagery with isometric opponens pollicis activity: influence of vision during motor imagery. NeuroRehabilitation 2014;34:725-9.

9. Kosslyn SM, Thompson WL, Sukel KE, Alpert NM. Two types of image generation: evidence from PET. Cogn Affect Behav Neurosci 2005;5:41-53.

10. Fukumoto Y, Bunno Y, Suzuki T. Effect of motor imagery on excitability of spinal neural function and its impact on the accuracy of movement-considering the point at which subjects subjectively determine the 50\%MVC point. J Phys Ther Sci 2016; 28:3416-20.

11. Sugata H, Hirata M, Yanagisawa T, Matsushita K, Yorifuji S, Yoshimine T. Common neural correlates of real and imagined movements contributing to the performance of brain-machine interfaces. Sci Rep 2016;6:24663.

12. Hahn N, Jansen P, Heil M. Preschoolers' mental rotation: sex differences in hemispheric asymmetry. J Cogn Neurosci 2010;22: 1244-50.

13. Zacks JM. Neuroimaging studies of mental rotation: a meta-analysis and review. J Cogn Neurosci 2008;20:1-19.

14. Cona G, Panozzo G, Semenza C. The role of dorsal premotor cor- 
tex in mental rotation: a transcranial magnetic stimulation study. Brain Cogn 2017;116:71-8.

15. Schendan HE, Stern CE. Mental rotation and object categorization share a common network of prefrontal and dorsal and ventral regions of posterior cortex. Neuroimage 2007;35:1264-77.

16. Alivisatos B. The role of the frontal cortex in the use of advance information in a mental rotation paradigm. Neuropsychologia 1992;30:145-59.

17. Barnes J, Howard RJ, Senior C, Brammer M, Bullmore ET, Simmons A, et al. Cortical activity during rotational and linear transformations. Neuropsychologia 2000;38:1148-56.

18. Harris IM, Egan GF, Sonkkila C, Tochon-Danguy HJ, Paxinos G, Watson JD. Selective right parietal lobe activation during mental rotation: a parametric PET study. Brain 2000;123:65-73.

19. Peters M, Lehmann W, Takahira S, Takeuchi Y, Jordan K. Mental rotation test performance in four cross-cultural samples $(n=$
3367): overall sex differences and the role of academic program in performance. Cortex 2006;42:1005-14.

20. Feredoes EA, Sachdev PS. Differential effects of transcranial magnetic stimulation of left and right posterior parietal cortex on mental rotation tasks. Cortex 2006;42:750-4.

21. Williams JD, Rippon G, Stone BM, Annett J. Psychophysiological correlates of dynamic imagery. Br J Psychol 1995;86(Pt 2): 283-300.

22. Wang Z, Guo X, Lyu Y, Chen H, Tong S. Spatiotemporal differences of brain activation between internal and external strategies in mental rotation: a behavioral and ERD/ERS study. Neurosci Lett 2016;623:1-6.

23. Bhattacharya J, Petsche H, Feldmann U, Rescher B. EEG gamma-band phase synchronization between posterior and frontal cortex during mental rotation in humans. Neurosci Lett 2001; 311:29-32. 